\title{
Research Paper \\ Effects of Three Types of Massage on Serum Levels of Malondialdehyde, Super- oxide Dismutase and Glutathione Peroxidase After One Session of Exhaustive Exercise in Female Futsal Players
}

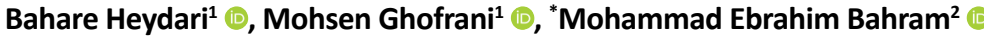

1. Department of Physical Education and Sport Sciences, Faculty of Educational Sciences and Psychology, University of Sistan and Baluchestan, Zahedan, Iran 2. Department of Physical Education and Sport Sciences, Faculty of Educational Sciences and Psychology, University of Mohaghegh Ardabili, Ardabil, Iran.

\begin{tabular}{|c|c|}
\hline $\begin{array}{l}\text { Use your device to scan } \\
\text { and read the article online }\end{array}$ & dtation: Heydari B, Ghofrani M, Bahram ME. [Effects of Three Types of Massage on Serum Levels of Malondialdehyde, \\
\hline arita & Superoxide Dismutase and Glutathione Peroxidase After One Session of Exhaustive Exercise in Female Futsal Players (Persian)]. \\
\hline Fisticis & Complementary Medicine Journal. 2021; 10(4):328-339. https://doi.org/10.32598/cmja.10.4.631.2 \\
\hline Prifits & doil'https://doi.org/10.32598/cmja.10.4.631.2 \\
\hline
\end{tabular}

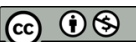

Article Info:

Received: 11 Oct 2020 Accepted: 06 Nov 2020 Available Online: 01 Jan 202

Keywords:

Massage, Malondialdehyde, Glutathione peroxidase, Superoxide dismutase, Exhaustive exercise

\section{A B STRACT}

Objective The production of reactive oxygen species in exercise causes oxidative stress which disturbs the balance of oxidants and antioxidants, causing destructive effects on cells. The present study aims to investigate the effect of three types of massage (Swedish, Russian, Thai) on serum levels of Malondialdehyde (MDA), Glutathione Peroxidase (GPX) and Superoxide Dismutase (SOD) following one session of exhaustive exercise.

Methods This quasi-experimental study was conducted on 48 female futsal players aged 17-22 years in Zahedan, Iran who were selected using a purposive sampling method, and randomly divided into four groups of Swedish massage (Long strokes with pressing and tapping using hands), Russian massage (Medium to high pressure), Thai massage (Pressure to certain parts of the body) and Control. The exercise program was based on Bruce protocol. Serum levels of MDA, GPX and SOD were measured by before and immediately after exercise and after massage. Data analysis was performed using repeated measures ANOVA, considering a significance level of $P \leq 0.05$.

Results In all three types of massage, there was a significant decrease in serum level of MDA $(0.22 \pm 0.08)$, and a significant increase in GPX $(1.84 \pm 0.46)$ and SOD $(10.02 \pm 2.86)$ levels after exhaustive $(P=0.001)$. No significant difference was observed in the control group.

Conclusion It seems that Russian, Thai, and Swedish types of massage can affect the serum levels of the MDA (as an oxidative stress marker) and the antioxidant enzymes of GPX and SOD during the postexercise recovery period.

\section{Extended Abstract}

\section{Introduction}

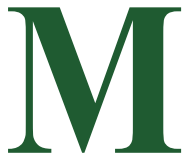

alondialdehyde (MDA) is a small but stable product of the lipid peroxidation that is resulted from the breakdown of unstable peroxides of unsaturated fatty acids
[3] Given that intense exercise causes oxidative stress, cells use the enzymatic antioxidant defense system including Glutathione Peroxidase (GPX) and Superoxide Dismutase (SOD) as the first line of defense against oxidative stress. Massage includes a group of manual techniques on body tissues to affect the neuromuscular, skin, and joints systems, reduce stress and pain, and improve the blood and lymphatic circulation. It has long been used as a complementary

\section{* Corresponding Author:}

Mohammad Ebrahim Bahram, PhD.

Address: Department of Physical Education and Sport Sciences, Faculty of Educational Sciences and Psychology, University of Mohaghegh Ardabili, Ardabil, Iran. Tel: +98 (913) 2627940

E-mail: bahramsport2010@gmail.com 
method for the treatment and improvement of body function and the prevention of injuries [10].

To our knowledge, no studies have been conducted on the effect of massage after exercise on the MDA, SOD and GPX markers. However, there are some studies on the effect of various massages on the markers of muscle injury, oxidation, anti-oxidation, and immune system. Yousefi et al. showed that massage after intense physical activity had a positive effect on immunoglobulin A and neutrophils in athletes with disabilities [14]. Naghizadeh et al. reported that the serum levels of creatinine kinase and lactate dehydrogenase slightly increased after massage compared to an eccentric exercise session [15]. Sadat and Hosseinizadeh in a study investigated the effect of sport massage on hematological parameters in semi-professional male runners. Their results showed a significant difference between the two types of active and sports massage in white blood cell, hemoglobin and hematocrit levels [16]. It has also been reported that massage has no effect on the strength and athletic performance and may even cause a decline in athletic performance [19]. Considering the importance of the massage, the present study aims to evaluate the effect of three types of massage on serum levels of MDA, SOD and GPX following a session of exhaustive exercise.

\section{Materials and Methods}

This is a quasi-experimental study conducted on 48 female futsal players aged 17-22 years in Zahedan, Iran during 2019-2020, who were voluntarily participated and randomly divided into four groups: Swedish massage, Russian massage, Thai massage, and control. Five cc of fasting blood was taken from the anterior brachial vein of subjects three times simultaneously from 8 to 10 A.M. To examine the difference within groups, repeated measures ANOVA was used and to measure differences between groups, one-way ANOVA was carried out. The significance level was set at 0.05 .

\section{Results}

As shown in Table 1, the results of repeated measures ANOVA showed that massage after exhaustive exercise leads to a significant reduction in MDA ( $\mathrm{F}=73.811$, $\mathrm{P}=0.001)$, GPX $(\mathrm{F}=44.237, \mathrm{P}=0.001)$ and $\mathrm{SOD}(\mathrm{F}=14.594$, $\mathrm{P}=0.001$ ) levels. The results of one-way ANOVA showed a significant difference between the effects of three types of sports massage on serum levels of MDA, SOD and GPX after one session of exhaustive exercise $(\mathrm{P}=0.001)$.

Table 1. Between-subjects and within-subjects effects tests (Repeated measures ANOVA)

\begin{tabular}{|c|c|c|c|c|c|c|}
\hline \multirow[b]{2}{*}{ Variables } & \multirow[b]{2}{*}{ Groups } & \multicolumn{3}{|c|}{ Mean $\pm S D$} & \multicolumn{2}{|c|}{ Sig. } \\
\hline & & Pre-exercise & After Massage & $\begin{array}{l}\text { Immediately } \\
\text { After Exercise }\end{array}$ & Within-subjects & Between-subjects \\
\hline \multirow{4}{*}{$\begin{array}{c}\text { MDA } \\
\text { (nmol/mg protein) }\end{array}$} & Swedish massage & $0.63 \pm 0.12$ & $0.64 \pm 0.12$ & $0.41 \pm 0.04$ & $0.04 *$ & \multirow{4}{*}{$0.001^{*}$} \\
\hline & Russian massage & $0.61 \pm 0.10$ & $0.061 \pm 0.10$ & $0.48 \pm 0.05$ & $0.02 *$ & \\
\hline & Thai massage & $0.60 \pm 0.12$ & $0.61 \pm 0.12$ & $0.45 \pm 0.03$ & $0.03^{*}$ & \\
\hline & Control & $0.57 \pm 0.14$ & $0.56 \pm 0.15$ & $0.57 \pm 0.14$ & 0.26 & \\
\hline \multirow{4}{*}{$\begin{array}{c}\text { SOD } \\
(\mu \mathrm{m} / \mathrm{mg} \text { protein) }\end{array}$} & Swedish massage & $61.68 \pm 5.85$ & $61.66 \pm 4.84$ & $71.70 \pm 2.99$ & $0.02 *$ & \multirow{4}{*}{$0.001^{*}$} \\
\hline & Russian massage & $61.39 \pm 3.09$ & $61.38 \pm 3.09$ & $68.26 \pm 2.84$ & $0.002^{*}$ & \\
\hline & Thai massage & $51.86 \pm 4.19$ & $54.35 \pm 4.69$ & $65.29 \pm 2.86$ & $0.01^{*}$ & \\
\hline & Control & $55.03 \pm 4.05$ & $54.86 \pm 5.82$ & $51.36 \pm 5.42$ & 0.09 & \\
\hline \multirow{4}{*}{$\begin{array}{c}\text { GPX } \\
(\mu \mathrm{m} / \mathrm{mg} \text { protein) }\end{array}$} & Swedish massage & $2.54 \pm 0.92$ & $2.53 \pm 0.93$ & $4.38 \pm 4.38$ & $0.002 *$ & \multirow{4}{*}{$0.003^{*}$} \\
\hline & Russian massage & $2.74 \pm 0.87$ & $2.72 \pm 0.86$ & $3.24 \pm 0.10$ & $0.003^{*}$ & \\
\hline & Swedish massage & $3.20 \pm 0.92$ & $3.21 \pm 0.89$ & $0.041 \pm 0.04$ & $0.04^{*}$ & \\
\hline & Russian massage & $2.42 \pm 0.77$ & $2.44 \pm 0.71$ & $0.48 \pm 0.05$ & $0.02 *$ & \\
\hline
\end{tabular}

* The difference is significance at $\mathrm{P} \leq 0.05$. 


\section{Conclusion}

This study aimed to investigate the effect of three types of massage on serum levels of MDA, SOD and GPX following a session of exhaustive exercise. To the best of our knowledge, there is no other similar study to compare our findings with their results. Yousefi and Azamian in a study showed that 20 minutes of effleurage sports massage can have a beneficial effect on the immune system of wheelchair basketball players [14]. This is consistent with our results. There is a powerful antioxidant called cytochrome that recycles reactive oxygen species and produces water by transferring electrons to oxygen; it reduces electron emission and, thus, the production of reactive oxygen species, and electron leakage [22].

The findings of the present study are against the results of Ang et al. and Kim et al. [24, 25]. This discrepancy may be due to the type and technique of applied massage. Consistent with the present study, Piri et al. showed that massage therapy leads to a significant reduction in oxidation markers by $25 \%$ [26]. Against the present study, Billhall et al. showed that massage therapy has no effect on biochemical markers [32]. Due to impaired balance between the oxygen consumption and the oxygen required by the involved tissues and the creation of a process called ischemia, exhaustive exercise seems to cause damage to unsaturated lipids in tissue membranes and production of reactive oxygen species, which in turn stimulates lipid peroxidation and ultimately increases the production of free radicals.

It seems that the use of massage during the recovery period and rest after exhaustive exercise can reduce the level of MDA, as an oxidative stress marker, and increase oxidative enzymes of SOD and GPX.

\section{Ethical Considerations}

Compliance with ethical guidelines

This study was approved by the University of Sistan and Baluchestan (Code: IR.US.Rec.1399.31409). All participants in this study signed a written consent form and were assured of the confidentiality of their information.

\section{Funding}

This study was extracted from the MA. thesis of first author at the Department of Physical Education and Sports Sciences, University of Sistan and Baluchestan.
Authors' contributions

All authors contributed equally in preparing this article.

\section{Conflicts of interest}

The authors declared no conflict of interest. 


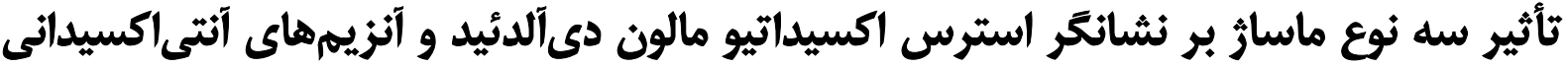

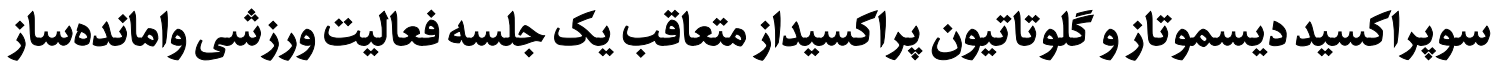

\author{
بهاره حيدرى' (ه، محسن غفرانى' ــ "محمدابراهيم بهرام'

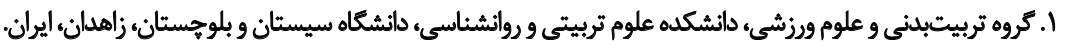

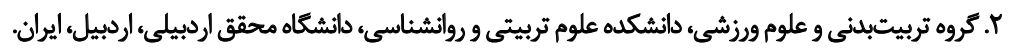

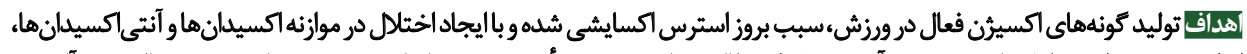

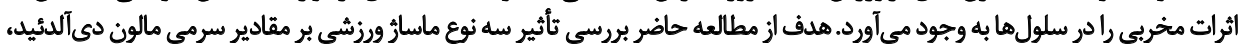

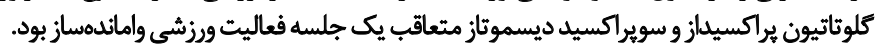

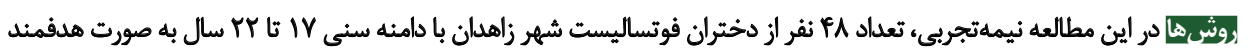

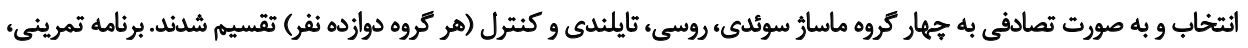

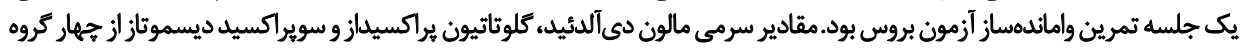

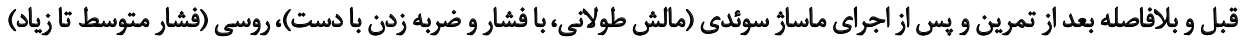

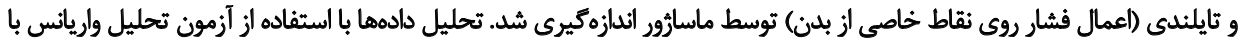

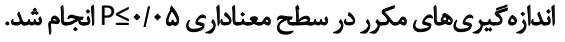

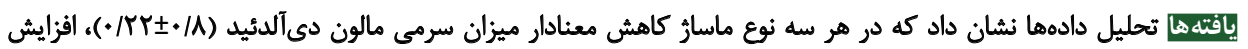

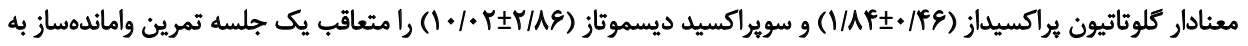

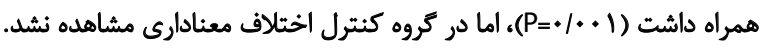

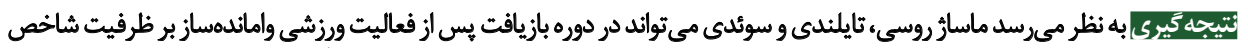

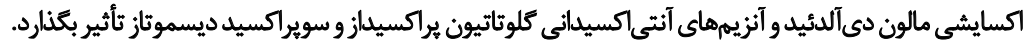

يراكسيداسيون ليخيدى است كه از تجزيه براكسيدهاى نايايدار

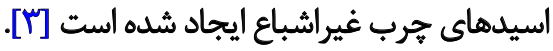

مقدمه

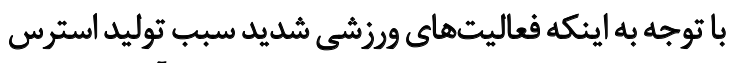

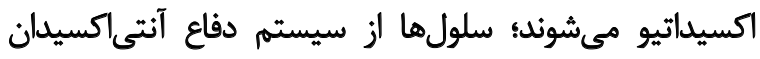

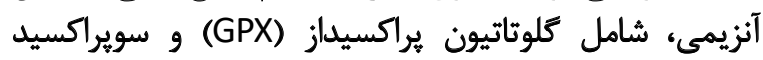

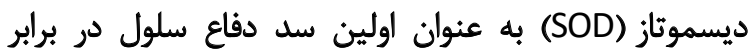
فشارهاى اكسايشى استفاده مي كنثن.

فشار اكسايشى مىتواند از طريق افزايش يراكسيداسيون

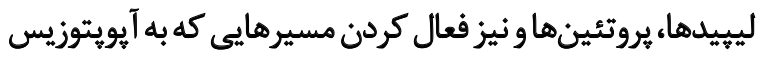

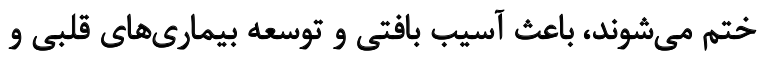

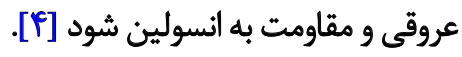
وجود راديكالهاى آزاد هنكام فعاليت ورزشى شديد، مصرف
فعاليت ورزشى باوجود فوايد كوناكونى كه براي سلامت عمومى

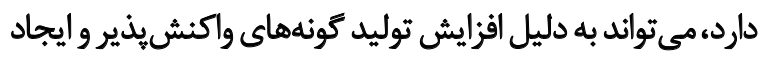

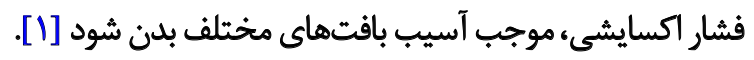

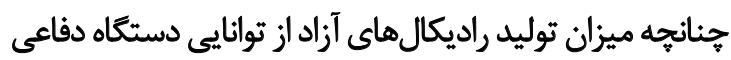

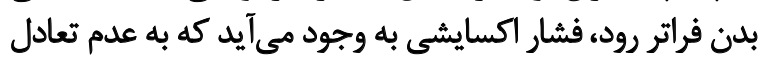

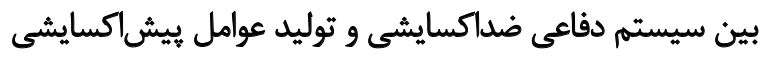

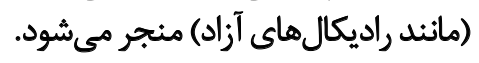

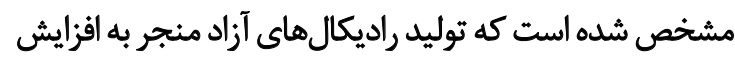

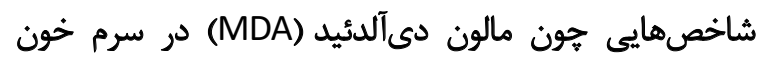

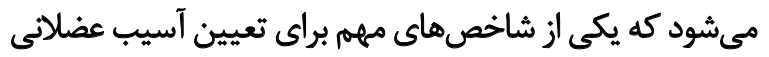

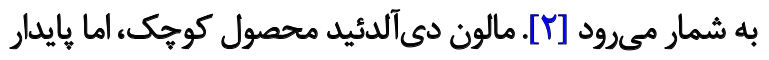


تحريك بذيرى عصبى و اضطراب. از نظر آنها، ماسار موجب بهبود

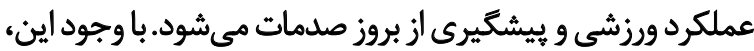

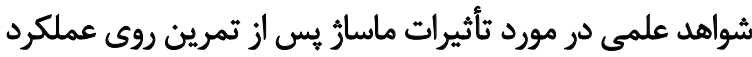

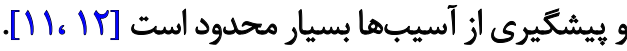

عقيده معمول بر آن است كه انجام ماهرانه ماسار موجب

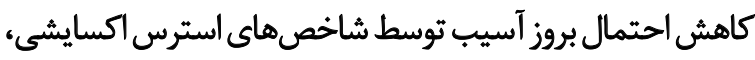

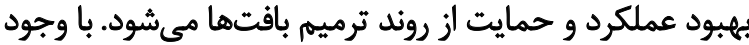

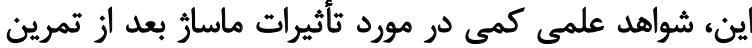

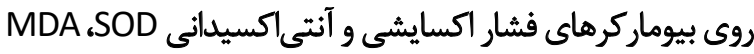

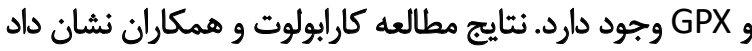

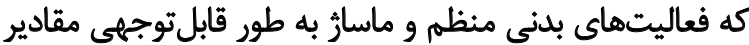

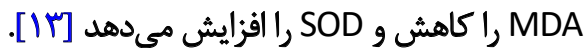
در اين راستا، مطالعاتى كه اثر سه نوع ماسار را بعد از تمرينات

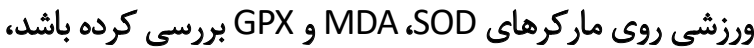
يافت نشد. باوجود اين، برخى از مطالعات اثر فعاليتهاى مختلف

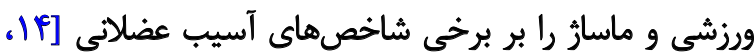

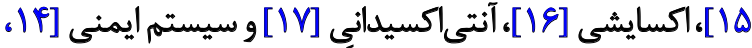

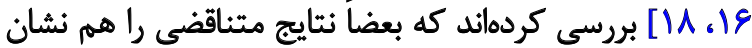

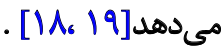

با توجه به اينكه ورزشكاران همواره در شرايط تمرين شديد

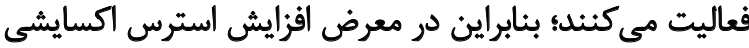

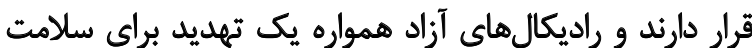

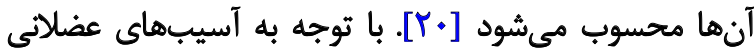

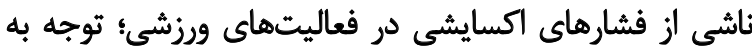

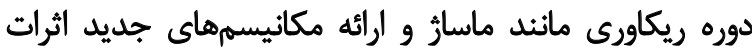
آنتى/كسيدانى ماساز ورزشى ضرورى به نظر مىرساند

همجنين، مطالعهاى در زمينه اثر ماسار بر شاخصهاى وجد اكسايشى و آنتىاكسيدانى SOD و از طرفى، مطالعاتى كه اثر ماسار بر آنزيمهاي آنسايشى مانئي

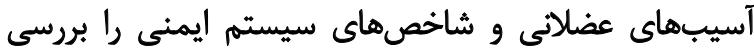

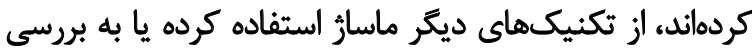

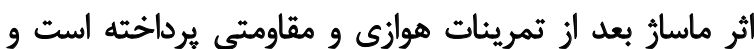

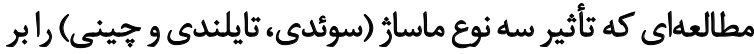

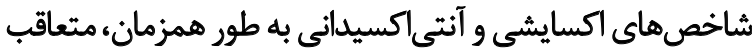

يك جلسه فعاليت وامائدهاز بررسى كرده باشد، يافت نشديد.

با توجه به اهميت موضوع، مطالعه حاضر در بيى باسخخ به اين

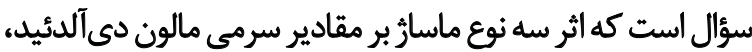
كلوتاتيون براكسيداز و سويراكسيد ديسموتاز متعاقب يك جائ جلسه فعاليت ورزشى واماندهاز جيست.
اكسيرن را به بيش از بيست برابر زمان استراحت افزايش مى دهاند

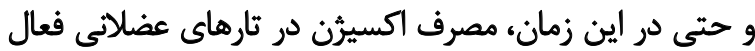

ممكن است به دويست برابر برسد [هـ].

هنكام آسيب بافت و غشاء عضلاني، محتويات آنه به خصوص

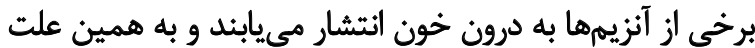

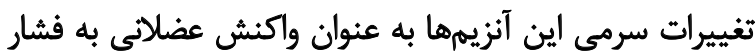

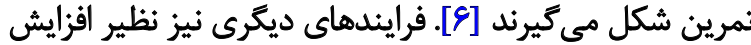

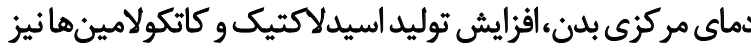

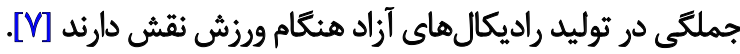

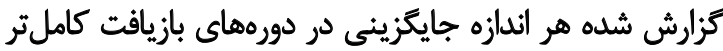

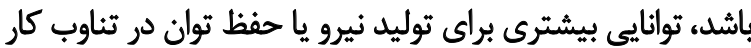

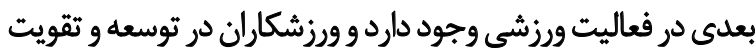

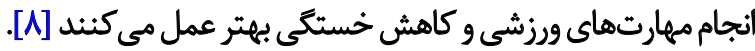
به عقيده بسيارى از صاحبنظران ورزشى، نوع فعاليت در

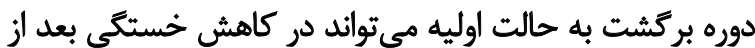

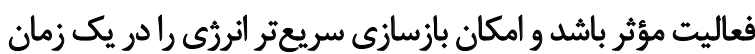

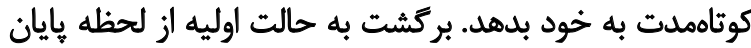

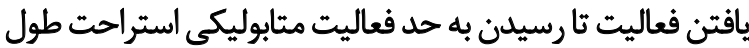

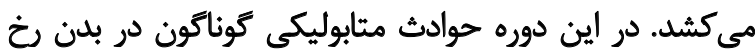

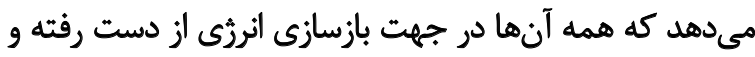

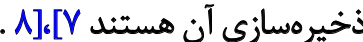

امروزه ماسار جزء لاينفك برنامه ورزشى ورزشكاران جهت

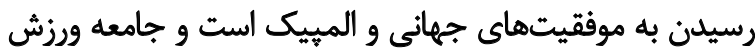

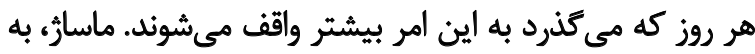

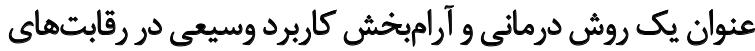

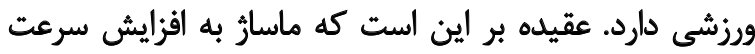

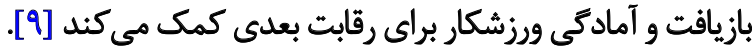
ماساز عبارث است از يك سرى حركات منظم، مرتب و اصولى

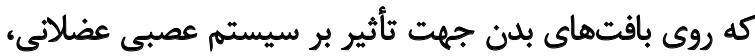

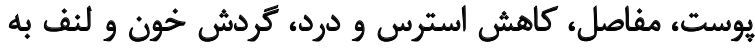

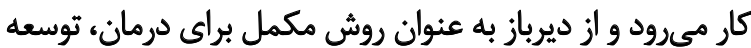
عملكرد و ييشيرى از وقوع صدمات استفاده مى شيده است دران.

در جوامع غربي يزشكان (r) درصد) ماسار را به عنوان يك

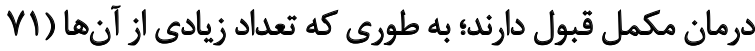

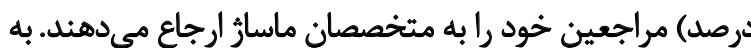

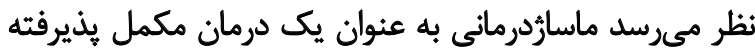

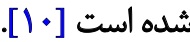

بسيارى از مربيان، ورزشكاران و درمانكران ورزشى بر اساس

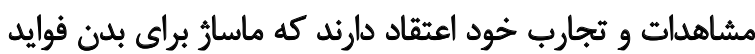

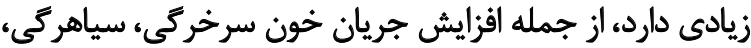

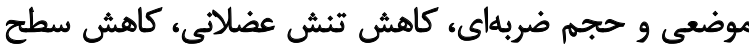


(يس آزمون Y)، بلافاصله بعد از ماسارً از آزمودنىهاصورت ترفت. اندازمكيرى ميزان مالون دى آلدئيد به روش الايزا، توسط

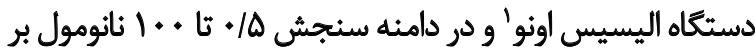

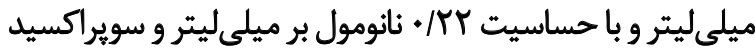

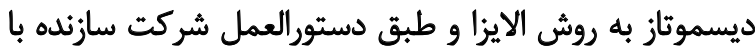

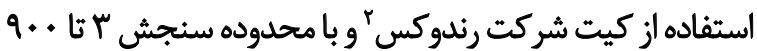

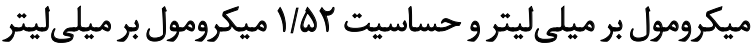

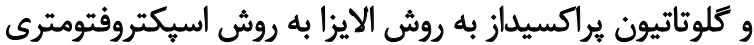

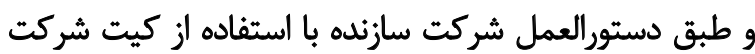

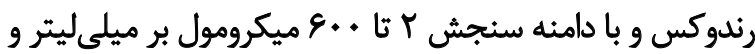

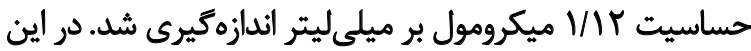

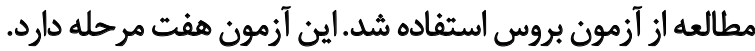

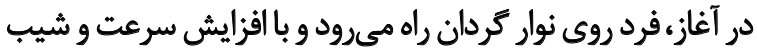

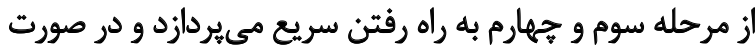
توانايى براي ادامه فعاليت، ورزشكار شروع به دويدن مئ كئد هر مرحله از آزمون بروس سه دقيقه طول مي كشد و شيب و

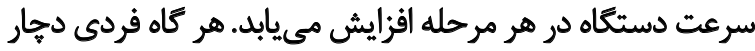

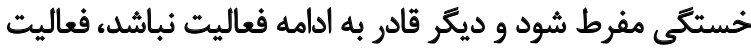

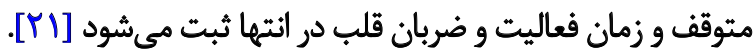
در تجزيه و تحليل آمارى، ابتدا تمامى مثغيرهاى كمّى توسط

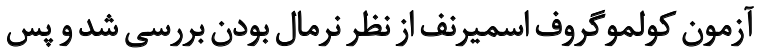

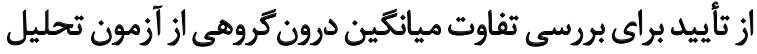

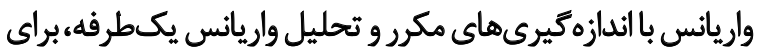
اندازهيرى تفاوتهاى بين گروهى استفاده شد.

با توجه به همكنى واريانسها و سطح معنادارى بالا از آزمون

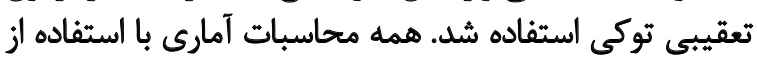

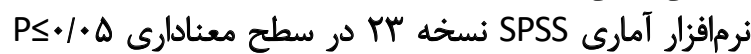

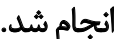

يافتهها

بين متغيرهاى سن، وزن و قد آزمودنى هاى شركت كنينده در

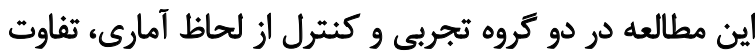

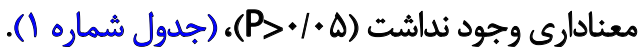

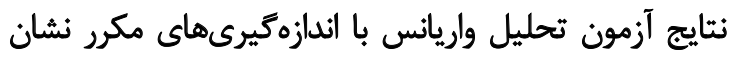

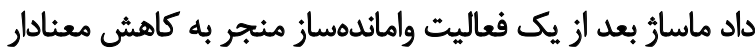

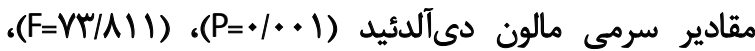

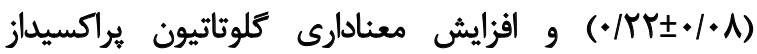

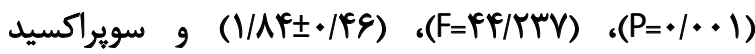

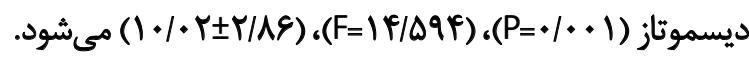

1. Alysys Uno

2. Randox

\section{مواد و روشىها}

اين يُروهش از نوع نيمهتجربى با كروههاى تجربى و كنترل بود.

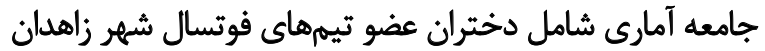

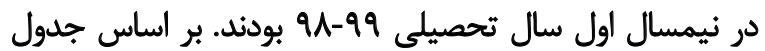

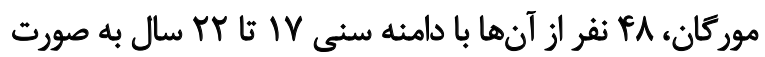

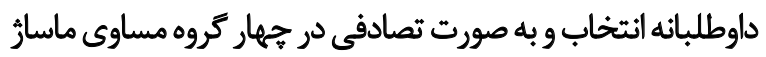

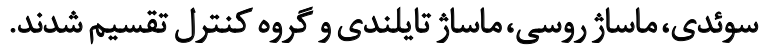
شرايط ورود به مطالعه شامل: ا- ورزشكار باشد با ب- نداشتن

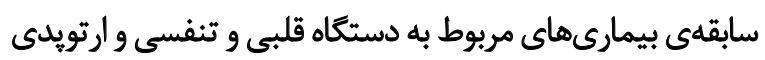

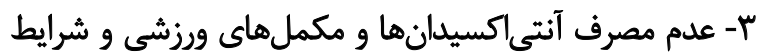

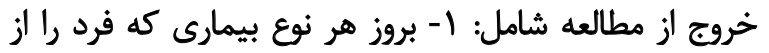

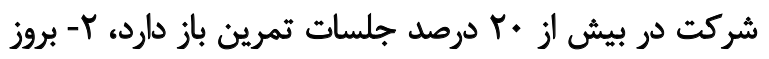

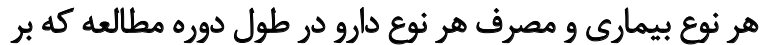

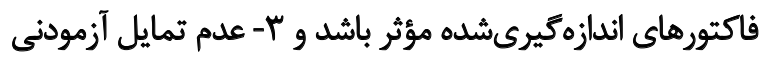

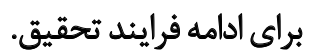

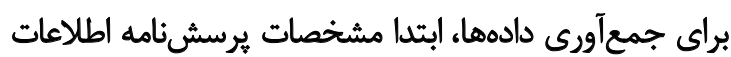

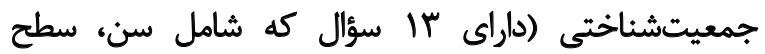

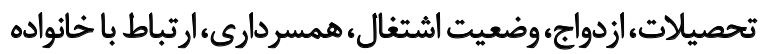

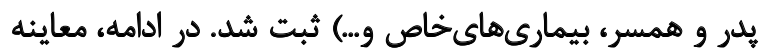

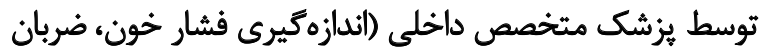

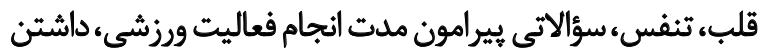

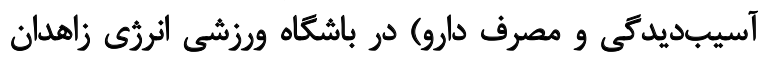

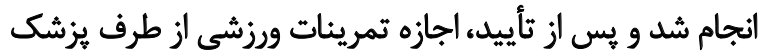
صادر شد.

قبل از شروع برنامه تمرينى، هدف از اجراى مطالعه براى

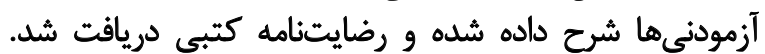

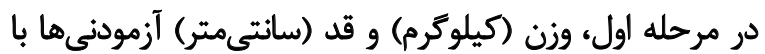

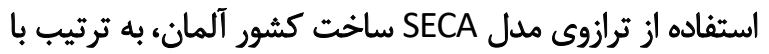

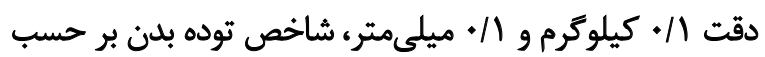
وزن تقسيم بر مجذور قد (كيلوكرم بر مترمربع) اندازه كيرى شد شد. درنهايت يس از هماهنكى با آزمودنىهاء از آزمودنى هاي هر

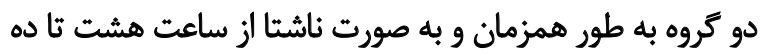

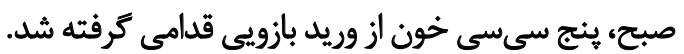
در طى اين يثروهش، سه بار از سه كروه ماسار تايلندى (اعمال

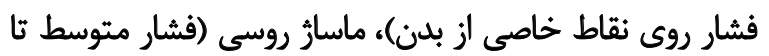

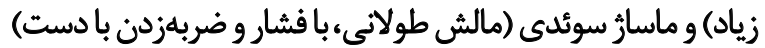

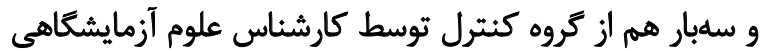

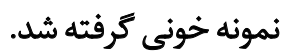

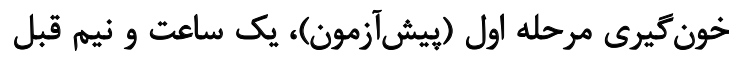

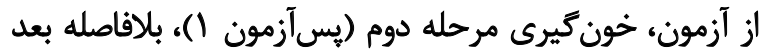
از تمرين واماندهاز (آزمون بروسي دوم و خونكيرى مرحله سوم بلافاصله بعد 
جدول ا. ويزُّكى هاي توصيفى آزمودنى ها در كروهاي موردمطالعه

\begin{tabular}{|c|c|c|c|c|}
\hline \multicolumn{4}{|c|}{ ميانغين+|نحراف معيار } & \multirow{2}{*}{ مثغير } \\
\hline كروه كتترل & كروه ماسار تايلندى & كروه ماساؤ روسى & كروه ماسار سوئدى & \\
\hline WTHET/TY & $r / \cdot . \pm Y / M Y$ & $19 / \Delta A \pm Y / A P$ & $19 / 8= \pm r / e F$ & سن (سال) \\
\hline $\mathscr{R} / N \Delta \pm \Delta / \Delta \Delta$ & $g \Delta / H \Psi T / R T$ & $E \Delta / \cdot A \pm F / P A$ & 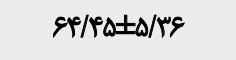 & وزن (كيلوكرم) \\
\hline $\mid V Q / P I \pm Y / N E$ & $\mid A T / M V \pm S / M$ & $M \cdot / r \Delta \pm r / q$. & 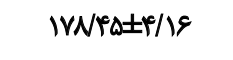 & قد (سانتىمتر) \\
\hline $1 Y / 9 \cdot \pm \cdot / 9 \Delta$ & $\mid w / r \cdot \pm 1 / r e$ & $M / A \cdot \pm \cdot / A \Delta$ & $\mid m / 1 \cdot \pm 1 / \cdot 8$ & جربى بلن (درصد) \\
\hline$r / \Delta \cdot \pm V / T r$ & $r M / r \cdot \pm r / r$. & $r r / T+ \pm 1 / r \Delta$ & $M T / T+ \pm I / \Delta C$ & $\left(\mathrm{~kg} / \mathrm{m}^{2}\right) \mathrm{BMI}$ \\
\hline
\end{tabular}

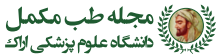

يسآزمون ا، اختلاف معنادارى مشاهده نشد. همجنين با توجه

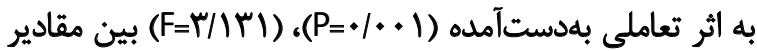

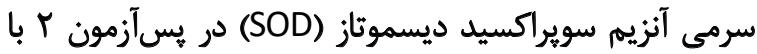

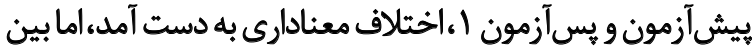

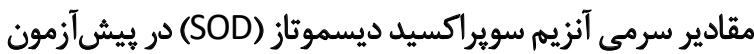

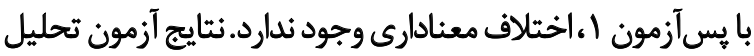

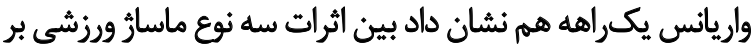

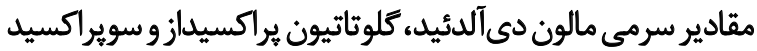

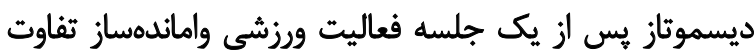

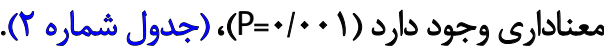

همجنين با توجه به اثر معنادارى تعامل فاكتور و زروه بر مقادير

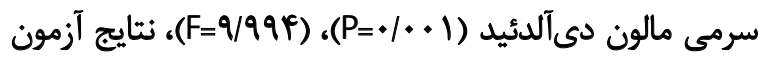

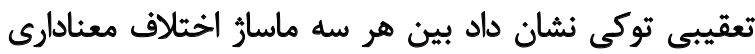

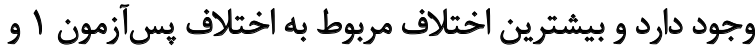
يسآزمون باست.

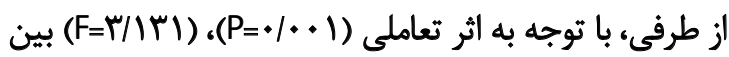

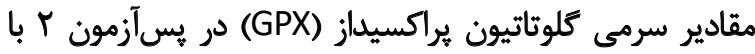

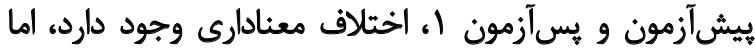

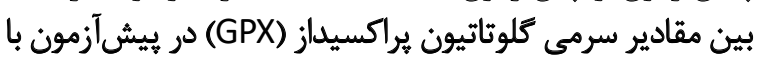

جدول ז. آزمون تحليل واريانس با اندازمكيرى مكرر (برسى اثر بينكروهى و درونكروهى)

\begin{tabular}{|c|c|c|c|c|c|c|}
\hline \multicolumn{2}{|c|}{ سطح معنادارى } & \multicolumn{3}{|c|}{ ميانكين+|نحرافمعيار } & \multirow{2}{*}{ آزمودنىها } & \multirow{2}{*}{ متغيرها } \\
\hline بين كَروهى & درون تروهى & بلافاصله بعد از تمرين & يسأزمون & ييش آزمون & & \\
\hline \multirow{4}{*}{$.1 .1^{\circ}$} & $\%$ & $. / 4)_{ \pm . * / . \varphi}$ & $. / 99 \pm . / / r$ & $. / R \pm . / r$ & ماسار سوثدى & \multirow{4}{*}{$\begin{array}{c}\text { MDA } \\
\text { (nmol/mg protein) }\end{array}$} \\
\hline & $\%+r^{\circ}$ & $. / F A \pm+/ . \Delta$ & $. / 91 \pm . / 1$ & $+|8| \pm \cdot 1 \mid$ & ماساز روسى & \\
\hline & $\%$ & $. / \% \Delta \pm . / . \psi$ & $\cdot|s| \pm \cdot / 1 r$ & $\cdot / 8 . \pm \cdot / 1 r$ & ماساز تايلندى & \\
\hline & . ME & $. / \Delta V \pm . / 1 P$ & $\cdot / \Delta \& \pm \cdot / 1 \Delta$ & $\cdot / \Delta Y \pm . / 1 F$ & كتترل & \\
\hline \multirow{4}{*}{.. $.1^{\circ}$} & $\%$ & $V / V \cdot \pm r / 99$ & gV/gg士r/Af & \&//8A $\pm \Delta / A \Delta$ & ماسار سوثلدى & \multirow{4}{*}{$\begin{array}{c}\text { SOD } \\
(\mu \mathrm{m} / \mathrm{mg} \text { protein) }\end{array}$} \\
\hline & H.+Y" & SNYEIY/AP & $9 / / \% A \pm r / .9$ & $q V / r q \pm r / . q$ & ماسار روسى & \\
\hline & $.1 .1^{\circ}$ & $S \Delta / Y q \pm Y / N$ & $\Delta \varphi / r \Delta \pm \varphi / q q$ & $\Delta V / N \& \pm \% / 19$ & ماسار تايلندى & \\
\hline & .1 .9 & $\Delta I / R E \pm \Delta / P r$ & $\Delta F / N E \pm \Delta / A Y$ & $\Delta \Delta / \cdot \mu \pm \varphi / \cdot \Delta$ & كتترل & \\
\hline \multirow{4}{*}{$1 .+\mu$} & /..r* & 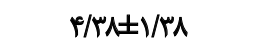 & $r / \Delta T \pm \cdot / 9 T$ & $r / \Delta F \pm \cdot / q T$ & ماسار سوتدى & \multirow{4}{*}{$\begin{array}{c}\text { GPX } \\
\text { ( } \mu \mathrm{m} / \mathrm{mg} \text { protein) }\end{array}$} \\
\hline & 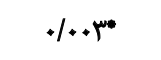 & $r / r \Psi \pm \cdot / 1$. & $T / M T \pm \cdot / N$ & $r / M \pm \pm / A Y$ & ماسارُ روسى & \\
\hline & $+|+\Delta|$ & $r / \Lambda) \pm \cdot / 11$ & $r / r \mid \pm . / 19$ & $r / r \cdot \pm . / 9 r$ & ماساز تايلندى & \\
\hline &.$/ 1 P$ & $T / T T \pm . N S$ & $r / P P \pm \cdot / M$ & $r / T T \pm \cdot / W$ & كتثرل & \\
\hline
\end{tabular}


افزايش فعاليت ياراسمياتيك شده و درنهايت منجر به افزايش

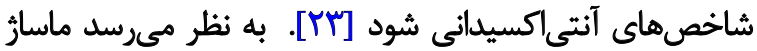

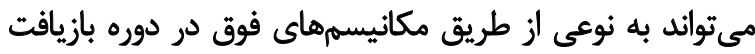

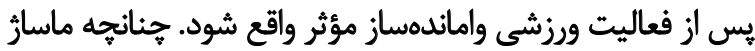

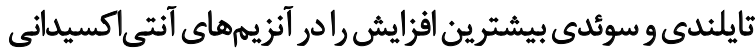
نشان دادند.از طرفى يافته مطالعه حاضر با مطالعات آنسات

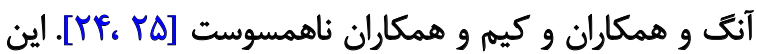

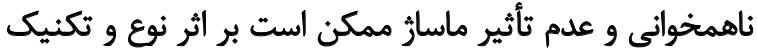

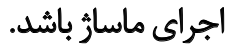

آنت، كيم و همكاران آنها در مطالعات خود از تكنيك ماساز

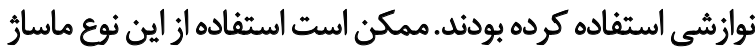

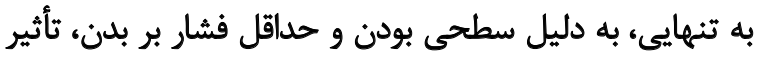

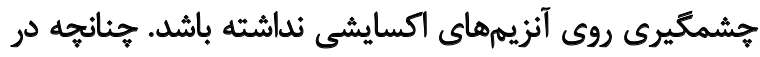

مطالعه حاضر از ماسار ثنايلندى، سوئدى و روسى استفادي ناده شد.

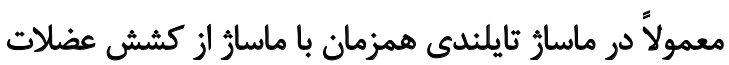

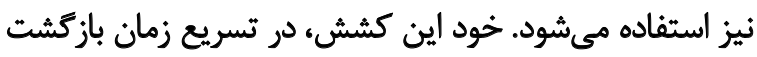

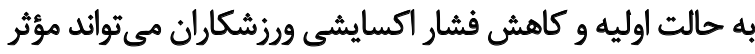

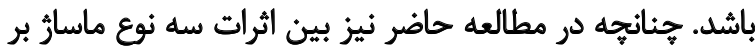

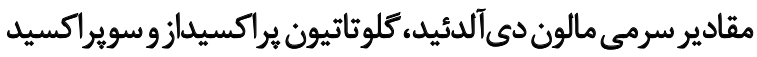

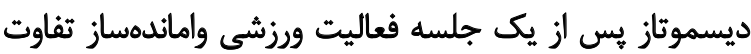

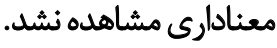

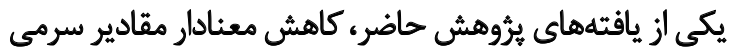

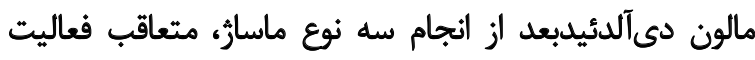

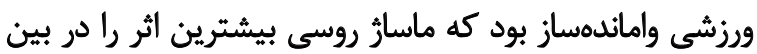

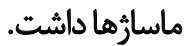

همسو با مطالعه حاضر ، بيرى و همكاران در مطالعه خود نشان

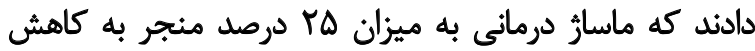

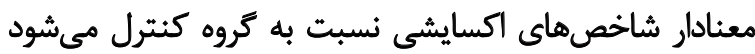

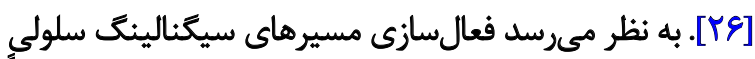

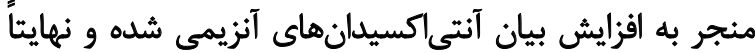

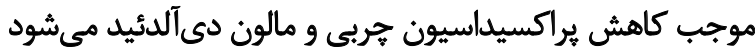

[YY]

همجنين ماسار به واسطه توانايىاش در به تحرك انداختن مايع

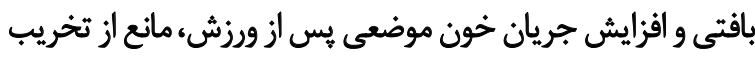

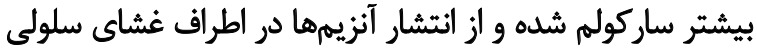

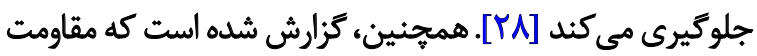

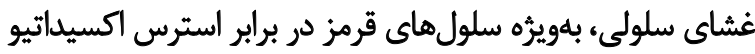

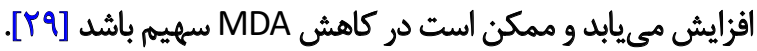

كزارش شده افرادى كه ورزش مي كنيند در مقايسه با همسن

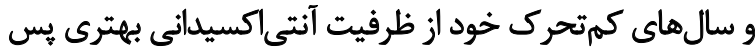

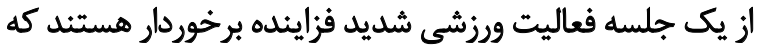
اين امر مىتواند ناشى از تعادل اكسيداني آنتى فاكسيداني مناسب
هدف از مطالعه حاضر بررسى اثر سه نوع ماسار بر مقادير سرمى

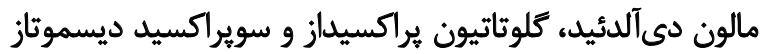
متعاقب يك جلسه فعاليت ورزشى واماندهساز بود.

نتايج مطالعه حاضر نشان داد كه انجام ماسار در سه نوع مختلف مادي

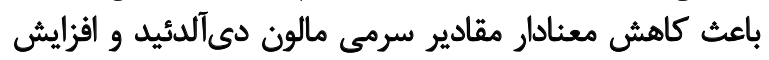

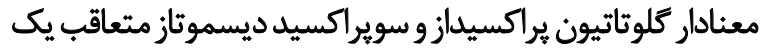
جلسه فعاليت واماندهساز در كروه تجريبى شدون

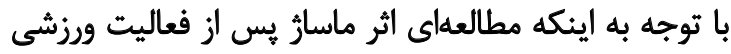

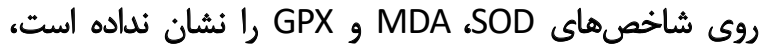

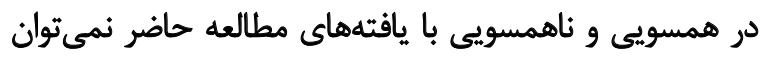

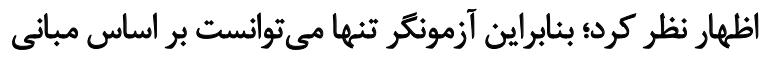

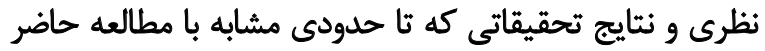

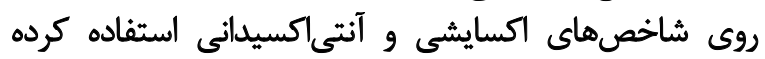

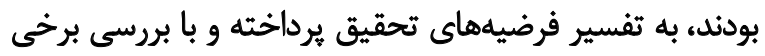

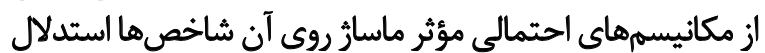
كند. نتايج مطالعه حاضر نشان داد كه ماسار يّ ازي فاز فعاليت

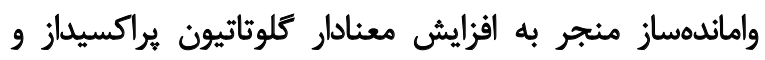

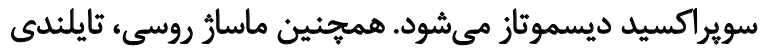
و سوئدى به ترتيب، بيشترين اثر راروى كاهش GPX g SOD

همراستا با مطالعه حاضر، يوسفى و اعظميان در مطالعهاي

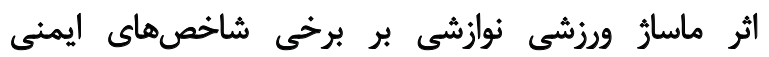

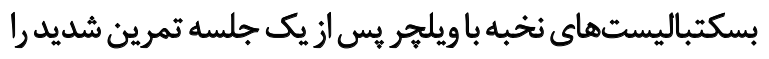

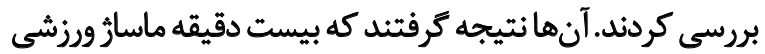

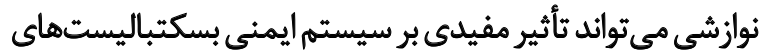

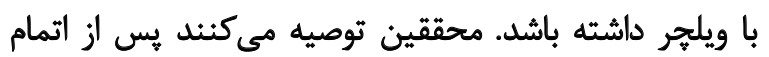

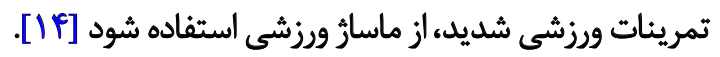

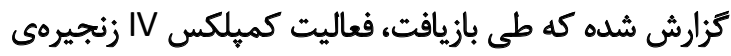

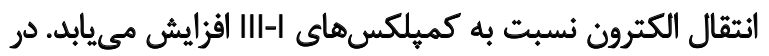

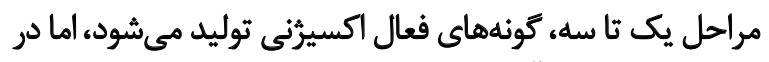

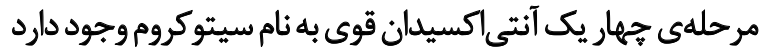

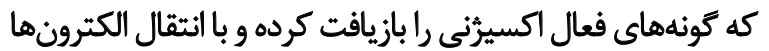

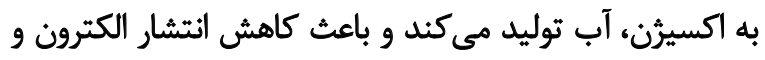

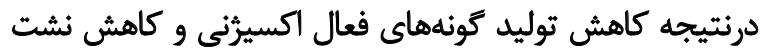

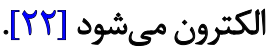

ازئ سازوكار احتمالى ديكر در افزايش شاخص إساى آنتى اكسيدانى

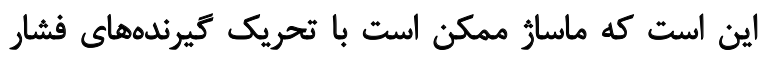

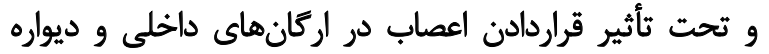

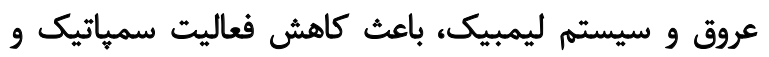




\section{ماحظات اخلاقى \\ ييروى أز اصول اخلاق بؤوهش}

كليه اصول اخلاقي در اين تحقيق مورد ثاييد كيمته

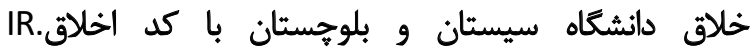

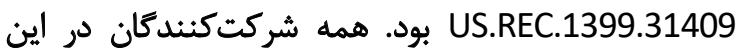

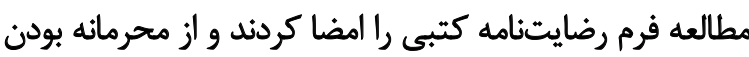
اطلاعات خود اطمينان داشتئد.

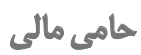

اين مقاله از ياياننامه كارشناسى ارشد نويسنده اول (بهاره

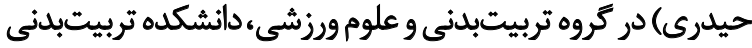

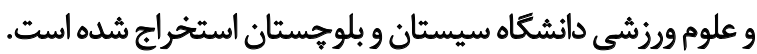

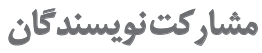

همه نويسند كان در همه زمينهها به طور مساوى مشاركت داشتند.

$$
\text { تعارض مئافع }
$$

نويسندكان مقاله هيجَّونه تعارضى در منافع اعلام نكردند.
ورزشكاران در مقايسه با غيرورزشكاران باشد [•"r]. همجنين

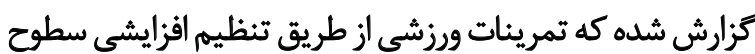

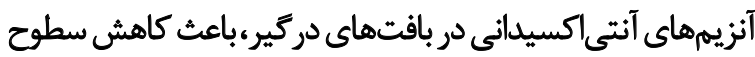

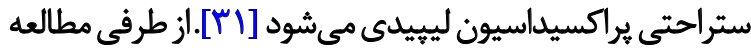

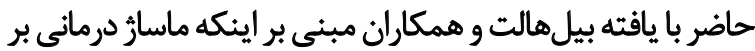

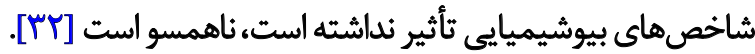

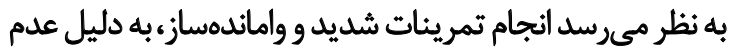

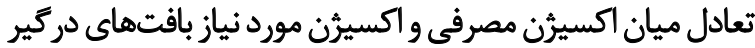

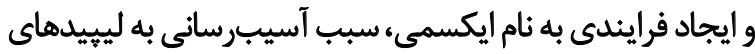

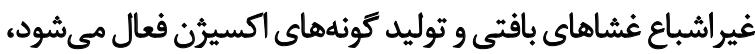

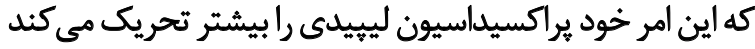

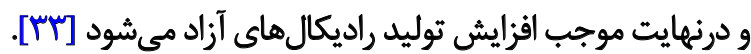

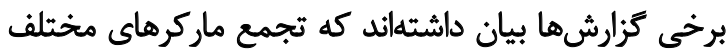

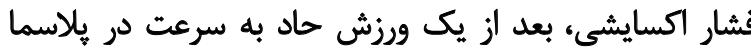

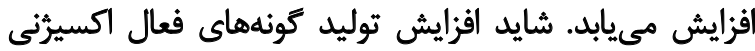

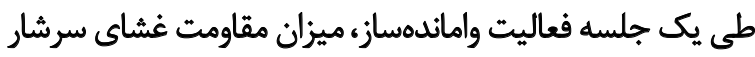

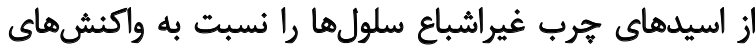

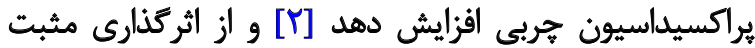
فيزيولوزيكى ماسارهها جلوكيرى كند.

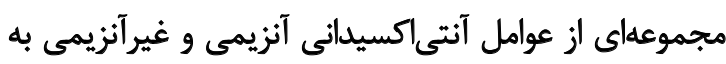

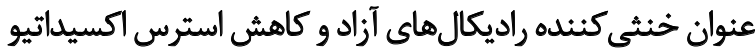

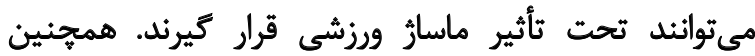

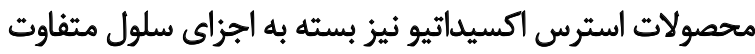

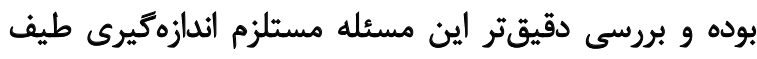

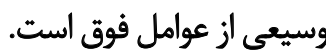

اين مطالعه جزء مطالعاتى است كه براى اولينبار اثر ماسار را

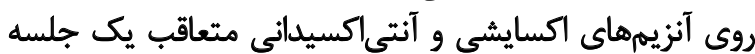

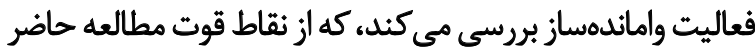

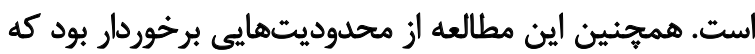

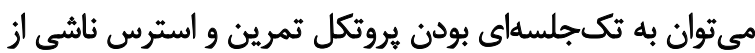
خون كيرى روى آزمودنى ها اشاره كرد. نتيجهنيرى

با توجه به نتايج اين مطالعه، به نظر ميرسد استفاده از براز

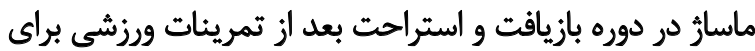

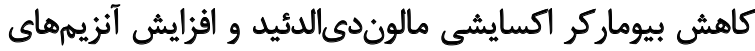

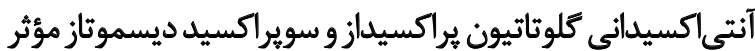

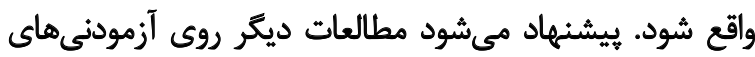

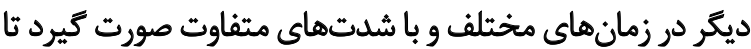
با قاطعيت بيشترى بتوان اظهار نظر كرد. 


\section{References}

[1] Alikhani S, Sheikholeslami-Vatani D. Oxidative stress and antioxidant responses to regular resistance training in young and older adult women. Geriatrics \& Gerontology International. 2019; 19(5):419-22. [DOI:10.1111/ggi.13636.] [PMID]

[2] McLeay Y, Stannard S, Houltham S, Starck C. Dietary thiols in exercise: Oxidative stress defence, exercise performance, and adaptation. Journal of the International Society of Sports Nutrition. 2017; 14(1):12-27. [DOI:10.1186/s12970-017-0168-9.] [PMID] [PMCID]

[3] Thirumalai T, Therasa SV, Elumalai EK and David E. Intense and exhaustive exercise induce oxidative stress in skeletal muscle. Asian Pacific Journal of Tropical Disease. 2011; 1(1):63-6. [DOI:10.1016/ S2222-1808 (11)60016-9]

[4] Belviranl M, Gökbel H, Okudan N, Başaralı K. Effects of grape seed extract supplementation on exercise-induced oxidative stress in rats. The British Journal of Nutrition. 2012; 108(2):249-56. [DOI:10.1017/ S0007114511005496] [PMID]

[5] Ahmadi-kakavandi M, Azizbeigi K, Qeysari SF. [The effects of progressive resistance training on malondialdehyde concentration and superoxide dismutase enzyme activity in inactive elderly women (Persian)]. Payavard. 2019; 13(2):151-9. http://payavard.tums.ac.ir/ article-1-6764-en.html

[6] Li S, Tan H, Wang N, Zhang Z, Lao L, Wong. The role of oxidative stress and antioxidants in liver diseases. International Journal of Molecular Sciences. 2015; 16(11):26087-124. [DOI:10.3390/ijms161125942] [PMID] [PMCID]

[7] Kostaropoulosia IA, Nikolaidis MG, Jamurtas AZ, Ikonomou GV Makrygiannis V, Papadopoulos $\mathrm{G}$, et al. Comparison of the blood redox status between long distance and short distance runners. Physiological Research. 2006; 55(6):611-6. [PMID]

[8] Heyman E, De Geus BA, Mertens I, Meeusen R. Effects of four recovery methods on repeated maximal rock climbing performance. Medicine \& Science in Sports \& Exercise. 2009; 41(6):1303-10. [DOI: 10.1249/MSS.0b013e318195107d]

[9] Hilbert JE, Asforzo G, Swensen T. Effect of massage on delayed onset muscle soreness. British Journal of Sports Medicine. 2003; 37(1):72-5. [DOI:10.1136/bjsm.37.1.72] [PMID] [PMCID]

[10] Buttagat V, Eungpinichpong W, Chatchawan U, Kharmwan S. The immediate effects of traditional Thai massage on heart rate variability and stress-related parameters in patients with back pain associated with myofascial trigger points. Journal of Bodywork and Movement Therapies. 2011; 15(1):15-23. [DOI:10.1016/j.jbmt.2009.06.005] [PMID]

[11] Pinar S, Kaya F, Bicer B, Erzeybek MS, Cotuk HB. Different recovery methods and muscle performance after exhausting exercise: Comparison of the effects of electrical muscle stimulation and massage. Biology of Sport. 2012; 29(4):269-75. [DOI:10.5604/20831862.1019664] [PMID] [PMCID]

[12] Weerapong P, Hume PA, Kolt GS. The mechanisms of massage and effects on performance, muscle recovery and injury prevention. Sports Medicine. 2005; 35(3):235-56. [DOI:10.2165/00007256200535030-00004] [PMID]

[13] Karabulut AB, Kafkas ME, Kafkas AS, Onal Y, Kiran TR. The effect of regular exercise and massage on oxidant and antioxidant parameters. Indian Journal of Physiology and Pharmacology. 2013; 57(4):378-83. [PMID]
[14] Yousefi Saghazi S, Azamian Jazi A. [The effect of caressing sports massage on some safety indicators of elite basketball players in wheelchairs after a hard training session (Persian)]. Journal of Exercise Science and Medicine. 2015; 7(2):267-78. [DOI:10.22059/JSMED.2015.56565]

[15] Emani E, MoshtaghEshgh Z, Hosseini T, Alavi Majd H, AbedSaeid $Z h$. [The effect of foot massage on physiological indicators of female patients with CVA admitted in the ICU (Persian)]. Journal of Shahid Sadoughi University of Medical Sciences. 2009; 17(2):76-82. https:// www.sid.ir/fa/journal/ViewPaper.aspx?id=93425

[16] Saadat F, Hossienzadeh M. [The effect of sports massage on hematological parameters in semi-professional male runners (Persian)]. Report of Health Care Journal. 2019; 5(3):8-13. http://jrhc.miau.ac.ir/ article_3501.html

[17] Poorbarzegar M, Minoonejad H, Seidi F, Mozafaripour E. [The immediate effect of sports massage on proprioception of knee and ankle joints in collegiate male athletes (Persian)]. Scientific Journal of Kurdistan University of Medical Sciences. 2017; 21(6):72-82. http:// sjku.muk.ac.ir/article-1-2825-en.htm

[18] Ilbeigi S, Ayubi Avaz M, Saghebjoo M, zardast M. [Acute effects of proprioception, massage and dynamic stretching warm up protocols on serum CK and LDH activity levels after one session of Plyometric training in male volleyball players (Persian)]. Koomesh. 2016; 17(2):393-402. http://koomeshjournal.semums.ac.ir/article1-2762-en.html

[19] Hunter AM, Watt JM, Watt V, Galloway SDR. Effect of lower limb massage on electromyography and force production of the knee extensors. British Journal of Sports Medicine. 2006; 40(2):114-8. [DOI:10.1136/bjsm.2005.019075] [PMID] [PMCID]

[20] Fattahi Bafghi A, Homaee H M, Azarbayjani M A. [Effects of high intensity interval training and curcumin supplement on antioxidant enzyme in heart tissue of diabetic rats (Persian)]. Iranian Journal of Diabetes and Obesity. 2016; 8(3):135-41. http://ijdo.ssu.ac.ir/ article-1-309-en.html

[21] Bahram ME, Ghofrani M, Pourvaghar M J, Arabpour H. [Comparing the effect of wet cupping with that of intensive aerobic workout on $\mathrm{HDL}$ and LDL levels in young male athletes (Persian)]. Complementary Medicine Journal Arak. 2018; 8(3):2330-40. http://cmja.arakmu. ac.ir/article-1-442-en.html

[22] Parise G, Phillips SM, Kaczor JJ, Tarnopolsky MA. Antioxidant enzyme activity is up-regulated after unilateral resistance exercise training in older adults. Free Radical Biology and Medicine. 2005 39(2):289-95. [DOI:10.1016/j.freeradbiomed.2005.03.024] [PMID]

[23] Hernandez-Reif M, Ironson G, Field T, Hurley J, Katz G, Diego M, et al. Breast cancer patients have improved immune and neuroendocrine functions following massage therapy. Journal of Psychosomatic Research. 2004; 57(1):45-52. [DOI:10.1016/S0022-3999(03)00500-2]

[24] Ang JY, Lua JL, Mathur A, Thomas R, Asmar BI, Savasan S, Buck S, et al. A randomized placebo-controlled trial of massage therapy on the immune system of preterm infants. Pediatrics. 2012; 130(6):1549-58 [DOI:10.1542/peds.2012-0196] [PMID] [PMCID]

[25] Kim JO. Kim IS. [Effects of aroma self-foot reflexology massage on stress and immune responses and fatigue in middle-aged women in rural areas (Korean)]. Journal of Korean Academy of Nursing. 2012; 42(5):709-18. [DOI:10.1542/peds.2012-0196] [PMID] [PMCID]

[26] Sheikh-Sarraf B, Azarbayjani M-A, Agha Alinejad H. The effect of aerobic interval training and massage therapy on c-reactive protein and cardiorespiratory fitness in cardiovascular patients after coronary artery bypass graft. Journal of Applied Sports Physiology. 2016; 4(1):9-16. DOI: 10.7508/aass.2016.01.003 
[27] Samjoo IA, Safdar A, Hamadeh MJ, Raha S. Tarnopolsky MA. The effect of endurance exercise on both skeletal muscle and systemic oxidative stress in previously sedentary obese men. Nutrition \& Diabetes. 2013; 3(9):e88. [DOI:10.1038/nutd.2013.30] [PMID] [PMCID]

[28] Zainuddin Z, Newton M, Sacco P, Nosaka K. Effects of massage on delayed-onset muscle soreness, swelling, and recovery of muscle function. Journal of Athletic Training. 2005; 40(3):174-80. [PMID]

[29] Petibois C, Deleris G. Erythrocytes adaptation to oxidative stress in endurance training. Archives of Medical Research. 2005; 36(5):524-31. [DOI:10.1016/j.arcmed.2005.03.047] [PMID]

[30] Ceci R, Beltran-Valls MR, Duranti G, Dimauro I, Quaranta F, Pittaluga $M$, et al. Oxidative stress responses to a graded maximal exercise test in older adults following explosive-type resistance training. Redox Biology. 2014; 2(1):65-72. [DOI:10.1016/j.redox.2013.12.004] [PMID] [PMCID]

[31] Vezzoli A, Pugliese L, Marzorati M, Serpiello FR, La Torre A, Porcelli $\mathrm{S}$. Time-course changes of oxidative stress response to high-intensity discontinuous training versus moderate-intensity continuous training in masters runners. PLoS One. 2014; 9(1):e87506. [DOI:10.1371/ journal.pone.0087506] [PMID] [PMCID]

[32] Billhult A, Lindholm C, Gunnarsson R, Stener-Victorin E. The effect of massage on immune function and stress in women with breast cancer: A randomized controlled trial. Autonomic Neuroscience: Basic \& Clinical. 2009; 150(1-2):111-5. [DOI:10.1016/j.autneu.2009.03.010] [PMID]

[33] Dabidi Roshan V, Moslehi-Najafabadi E. The effect of short-term vitamin $E$ supplementation on some indexes of sport performances and lipid per-oxidation in healthy men. World Journal of Sport Sciences. 2009; 2:75-81. https://www.sid.ir/en/journal/ViewPaper. aspx?ID=322834 
This Page Intentionally Left Blank 